PROCEEDINGS OF THE

AMERICAN MATHEMATICAL SOCIETY

Volume 131, Number 1, Pages 265-274

S 0002-9939(02)06701-1

Article electronically published on June 12, 2002

\title{
WEYL FORMULA FOR HYPOELLIPTIC OPERATORS OF SCHRÖDINGER TYPE
}

\author{
ERNESTO BUZANO AND ANDREA ZIGGIOTO
}

(Communicated by David S. Tartakoff)

\begin{abstract}
In this work we consider a general class of hypoelliptic operators, for which we give an estimate of the remainder of the so-called Weyl asymptotic formula for the eigenvalues.
\end{abstract}

\section{INTRODUCTION}

In this paper we deal with the problem of the asymptotic estimate of the counting function $\mathcal{N}(\tau)$ of a hypoelliptic operator in $\mathbb{R}^{n}$, with symbol $h(x, \xi)$ diverging at infinity, in terms of the volume $\mathcal{W}(\tau)$ of the set

$$
\left\{(x, \xi) \in \mathbb{R}^{n} \times \mathbb{R}^{n} \mid h(x, \xi) \leqslant \tau\right\} .
$$

Our starting point is the very general estimate of the relative remainder

$$
\frac{\mathcal{N}(\tau)-\mathcal{W}(\tau)}{\mathcal{W}(\tau)}
$$

obtained by Dencker in [5], who generalized Hörmander's papers [6] and [7] to locally temperate metrics. As already remarked in [2], Dencker's estimate is not always infinitesimal, as $\tau \rightarrow+\infty$.

In this work we identify a wide class of hypoelliptic operators, we call of Schrödinger type, for which we manage to give an estimate of the kind

$$
\mathcal{N}(\tau)=\mathcal{W}(\tau)\left(1+O\left(\tau^{-\epsilon}\right)\right), \quad \text { as } \tau \rightarrow \infty,
$$

for a suitable $\epsilon>0$.

We employ the following notation: given two functions $f, g: X \rightarrow \mathbb{R}$, and a subset $A \subset X$, we write

$$
f(x) \prec g(x), \quad \forall x \in A,
$$

if there exists a constant $C$ such that

$$
f(x) \leq C g(x), \quad \forall x \in A .
$$

Received by the editors September 4, 2001.

2000 Mathematics Subject Classification. Primary 35P20, 47B06.

(C)2002 American Mathematical Society 


\section{Asymptotic BeHAVior of THE SPECTRUM OF FORMALLY HYPOELLIPTIC OPERATORS ON $\mathbb{R}^{n}$}

We recall some results from [5] and [6].

Let

$$
\phi(x, \xi ; y, \eta)=\sum_{j=1}^{n} \xi_{j} y_{j}-x_{j} \eta_{j}
$$

be the standard symplectic form on $\mathbb{R}^{n} \times \mathbb{R}^{n}$. A Riemannian metric $g_{x, \xi}(y, \eta)$ on $\mathbb{R}^{n} \times \mathbb{R}^{n}$ is locally $\phi$ temperate if it is slowly varying and there exist two positive real numbers $r$ and $N$ and a slowly varying metric $G_{x}(y)$ on $\mathbb{R}^{n}$ such that

$$
G_{x}(y) \leqslant g_{x, \xi}(y, \eta),
$$

for all $(x, \xi),(y, \eta) \in \mathbb{R}^{n} \times \mathbb{R}^{n}$, and

$$
g_{x, \xi}(z, \zeta) \prec g_{y, \eta}(z, \zeta)\left(1+g_{x, \xi}^{\phi}(x-y, \xi-\eta)\right)^{N},
$$

for all $(x, \xi),(y, \eta),(z, \zeta) \in \mathbb{R}^{n} \times \mathbb{R}^{n}$ such that

$$
G_{x}(x-y) \leqslant r .
$$

The quadratic form $g_{x, \xi}^{\phi}(y, \eta)$ appearing in (II) is the dual metric

$$
g_{x, \xi}^{\phi}(y, \eta)=\sup \left\{(\phi(y, \eta ; z, \zeta))^{2} \mid g_{x, \xi}(z, \zeta)=1\right\} .
$$

Similarly we say that a positive function $m(x, \xi)$ is locally $\phi, g$ temperate if it is $g$ continuous (see [6]) and

$$
m(x, \xi) \prec m(y, \eta)\left(1+g_{x, \xi}^{\phi}(x-y, \xi-\eta)\right)^{N},
$$

for all $(x, \xi),(y, \eta) \in \mathbb{R}^{n} \times \mathbb{R}^{n}$ such that

$$
G_{x}(x-y) \leqslant r .
$$

Definition 1. A differential operator $h^{w}$ is formally hypoelliptid 1 if its Weyl symbol $h(x, \xi)$ satisfies the following hypotheses:

1) $h$ is a smooth function such that

$$
h(x, \xi) \neq 0, \quad \forall(x, \xi) \in \mathbb{R}^{n} \times \mathbb{R}^{n} ;
$$

2) there exists a locally $\phi$ temperate metric $g_{x, \xi}(y, \eta)$ on $\mathbb{R}^{n} \times \mathbb{R}^{n}$ such that $|h|$ is locally $\phi, g$ temperate and

$$
h \in S(|h|, g)
$$

where $S(|h|, g)$ denotes the class of symbols of Weyl-Hörmander (see [6] and [5]).

Remark. Under suitable hypotheses on $h$ (see [10, [1] and [4]) one can show that $h^{w}$ is globally hypoelliptic in the following sense:

$$
u \in \mathcal{S}^{\prime} \& h^{w}(u) \in \mathcal{S} \Longrightarrow u \in \mathcal{S},
$$

where $\mathcal{S}$ is the Schwartz class and $\mathcal{S}^{\prime}$ are the tempered distributions. This justifies the term formally hypoelliptic in the definition above.

\footnotetext{
${ }^{1}$ This definition is not to be confused with Definition 2.3 of Chapter III of [11].
} 
Theorem 1. Consider a real positive and formally hypoelliptic symbol $h \in S(h, g)$ and assume that there exists a positive real number $\gamma$ such that

$$
g_{x, \xi}(y, \eta) \prec h(x, \xi)^{-\gamma} g_{x, \xi}^{\phi}(y, \eta)
$$

for all $(x, \xi),(y, \eta) \in \mathbb{R}^{n} \times \mathbb{R}^{n}$. Then $h^{w}$ is semi-bounded from below and essentially self-adjoint in $L^{2}\left(\mathbb{R}^{n}\right)$.

Moreover, if

$$
h(x, \xi) \rightarrow+\infty, \quad \text { as }|x|+|\xi| \rightarrow+\infty,
$$

then the closure $H$ of $h^{w}$ in $L^{2}\left(\mathbb{R}^{n}\right)$ has discrete spectrum diverging to $+\infty$.

Proof. This is Proposition 6.1 of [5].

Thanks to this theorem, we can define the counting function of the operator $H$ :

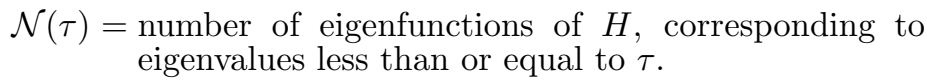

Theorem 2. Under the hypotheses of Theorem 1 assume there exists $\kappa>0$ such that

$$
h^{-\kappa} \in L^{1}
$$

Then for all $0<\delta<\gamma / 3$ we have

$$
\mathcal{N}(\tau)=\mathcal{W}(\tau)\{1+O(\mathcal{R}(\tau))\}, \quad \text { as } \tau \rightarrow+\infty
$$

where

$$
\mathcal{W}(\tau)=(2 \pi)^{-n} \int_{h \leqslant \tau} d x d \xi
$$

and

$$
\mathcal{R}(\tau)=\frac{\mathcal{W}\left(\tau+\tau^{1-\delta}\right)-\mathcal{W}\left(\tau-\tau^{1-\delta}\right)}{\mathcal{W}(\tau)}
$$

Remark. Estimate (7) is known as the Weyl formula.

Proof. If we change the hypothesis (6] with

$$
1+|x|+|\xi| \prec h(x, \xi)^{\kappa}, \quad \forall(x, \xi) \in \mathbb{R}^{n} \times \mathbb{R}^{n},
$$

this theorem becomes Theorem 6.2 of 5 .

A careful inspection of Dencker's argument shows that its proof continues to hold also under our more general hypothesis.

It is not always true that the remainder $\mathcal{R}(\tau)$ vanishes as $\tau \rightarrow+\infty$. In [2] there is an example of a Schrödinger operator with potential with algebraic growth at infinity, thus meeting in particular hypothesis (6), for which $\mathcal{R}(\tau)$ is unbounded as $\tau \rightarrow+\infty$. In the section which follows we estimate the remainder $\mathcal{R}(\tau)$ under rather general conditions. 


\section{Hypoelliptic differential operators of Schrödinger type AND ESTIMATE OF THE REMAINDER IN THE WEYL FORMULA}

Definition 2. A formally hypoelliptic differential operator $h^{w}$ is called of Schrödinger type if

$$
h(x, \xi)=p(\xi)+q(x)
$$

where $p$ is a real polynomial such that

$$
p(0)=0,
$$

and $q$ is a real-valued function, called a potential.

Theorem 3. Under the hypotheses of Theorem 1, consider a formally hypoelliptic operator of Schrödinger type $h^{w}=p^{w}+q$, and set

$$
\mathcal{V}(\tau)=\int_{q \leqslant \tau} d x .
$$

Assume that there exists $\tau_{0}>0$, such that

$$
\mathcal{V}(2 \tau) \prec \mathcal{V}(\tau), \quad \forall \tau \geqslant \tau_{0} .
$$

Then there exists $a>0$ such that for all $0<\epsilon<\frac{a}{1+a} \min \left\{1, \frac{\gamma}{3}\right\}$ we have

$$
\mathcal{N}(\tau)=\mathcal{W}(\tau)\left\{1+O\left(\tau^{-\epsilon}\right)\right\}, \quad \text { as } \tau \rightarrow+\infty .
$$

Remark. When $p$ is a multi-quasi-elliptic polynomial, one can compute the constant $a$; see [3].

Proof. First we show that hypothesis (6) is satisfied, so that Weyl formula (7) holds; then we estimate the remainder $\mathcal{R}(\tau)$.

Lemma 1. There exists $\kappa>0$ such that (6) is satisfied.

Proof. Since $h>0$, we have

$$
p(\xi)+q(x)>0, \quad \forall x, \xi \in \mathbb{R}^{n} .
$$

Because $p(0)=0$ and $q(x) \rightarrow+\infty$, as $|x| \rightarrow+\infty$, by (4), it follows that

$$
L=\inf _{x \in \mathbb{R}^{n}} q(x)>0 .
$$

Hence there exists $R>0$ such that

$$
p(\xi)+q(x) \geqslant \sqrt{p(\xi) q(x)}, \quad \forall \xi \notin B(R) \& \forall x \in \mathbb{R}^{n},
$$

where $B(R)$ is the open ball with center at the origin and radius $R$. Therefore it suffices to show that there exists $\kappa_{1}>0$ and $\kappa_{2}>0$ such that $p^{-\kappa_{1}} \in L^{1}\left(\mathbb{R}^{n} \backslash B(R)\right)$ and $q^{-\kappa_{2}} \in L^{1}\left(\mathbb{R}^{n}\right)$.

From (21) and (44) we have that $p \rightarrow+\infty$, as $|\xi| \rightarrow+\infty$. Then, because $p$ is a polynomial, it follows from the Tarski-Seidenberg Theorem that there exists $k_{0}>0$ such that

$$
p(\xi) \succ|\xi|^{k_{0}}, \quad \forall|\xi| \geqslant R .
$$

In fact,

$$
E=\left\{(s, t, \xi) \in \mathbb{R} \times \mathbb{R} \times\left.\mathbb{R}^{n}|s=| \xi\right|^{2} \& p(\xi)=t\right\}
$$


is algebraic in $\mathbb{R}^{2+n}$, and therefore

$$
f(s)=\inf _{|\xi|^{2}=s} p(\xi)=\inf \{t \mid \exists s \exists \xi((s, t, \xi) \in E)\}<+\infty
$$

is semi-algebraic. Moreover $f(s) \rightarrow+\infty$, as $s \rightarrow+\infty$; hence (14) follows from Corollary A.2.6 of [8].

It is clear that estimate (14) implies the existence of $\kappa_{1}$.

Now we prove the existence of $\kappa_{2}$. First we show that there exists $c_{0}>0 \mathrm{such}$ that

$$
\mathcal{V}(\tau) \prec \tau^{c_{0}}, \quad \forall \tau>0 .
$$

From (13) we have that $\mathcal{V}(\tau)=0$ for $\tau \leqslant L$, hence it suffices to prove (15) for $\tau \geqslant \tau_{0}>0$. From (11) we have that there exists $c>0$ such that

$$
\mathcal{V}(2 \tau) \leqslant c \mathcal{V}(\tau), \quad \forall \tau \geqslant \tau_{0} .
$$

Let $k_{1} \in \mathbb{N}$ be such that

$$
\tau_{0} \leqslant 2^{-k_{1}} \tau \leqslant 2 \tau_{0}
$$

By applying $k_{1}$ times inequality (16), we have

$$
\begin{aligned}
\mathcal{V}(\tau) & \leqslant c^{k_{1}} \mathcal{V}\left(2^{-k_{1}} \tau\right) \\
& \leqslant c^{\log _{2}\left(\tau / \tau_{0}\right)} \mathcal{V}\left(2 \tau_{0}\right) \\
& =\left(\frac{\tau}{\tau_{0}}\right)^{\log _{2} c} \mathcal{V}\left(2 \tau_{0}\right),
\end{aligned}
$$

which is 15. Now we prove the integrability of $q^{-\kappa_{2}}$ for $\kappa_{2}>c_{0}+1$. In fact, from (15) we have

$$
\begin{aligned}
\int q(x)^{-\kappa_{2}} d x & =\sum_{k=0}^{+\infty} \int_{k \leqslant q \leqslant k+1} q(x)^{-\kappa_{2}} d x \\
& \leqslant \int_{q \leqslant 1} q(x)^{-\kappa_{2}} d x+\sum_{k=1}^{+\infty} k^{-\kappa_{2}} \mathcal{V}(k+1) \\
& \prec \sum_{k=1}^{+\infty} k^{-\kappa_{2}}(k+1)^{c_{0}}<+\infty,
\end{aligned}
$$

for $\kappa_{2}>c_{0}+1$.

Thanks to Lemma 1 and Theorem 2 we have that (9) holds.

Now we recall the following result of Nilsson:

Theorem 4. The function

$$
\Phi(\lambda)=\int_{p(\xi) \leqslant \lambda} d \xi
$$

is a real analytic for $\lambda$ real and large and there exist a positive rational number a and a non-negative integer $b$, such that for all $k \in \mathbb{N}$ there exists $A_{k} \in \mathbb{R}$ such that the following estimate holds:

$$
\Phi^{(k)}(\lambda)=A_{k} \lambda^{a-k}(\log \lambda)^{b}(1+o(1)), \quad \text { as } \lambda \rightarrow+\infty .
$$


Proof. This is a special case of Theorem 3 of 9, where the author only assumes that $p$ is a polynomial such that $p(\xi) \rightarrow+\infty$, as $|\xi| \rightarrow+\infty$.

Let us draw some consequences from estimate (17):

Corollary 1. There exists $\lambda_{0}>0$ and $A>0$ such that

$$
\Phi\left(\lambda_{2}\right) \leqslant \Phi\left(\lambda_{1}\right)\left(\frac{\lambda_{2}}{\lambda_{1}}\right)^{A}, \quad \forall \lambda_{2} \geqslant \lambda_{1} \geqslant \lambda_{0} .
$$

Proof. Because $\Phi(\lambda)$ is positive and increasing, it is clear that in the estimate (17) we must have $A_{0}, A_{1}>0$. In particular, from (17) there exists $\lambda_{0}>0$ such that

$$
\Phi(\lambda) \geqslant \frac{A_{0}}{2} \lambda^{a}(\log \lambda)^{b}
$$

and

$$
\Phi^{\prime}(\lambda) \leqslant \frac{3 A_{1}}{2} \lambda^{a-1}(\log \lambda)^{b},
$$

for all $\lambda \geqslant \lambda_{0}$. It follows that

$$
\frac{\Phi^{\prime}(\lambda)}{\Phi(\lambda)} \leqslant \frac{3 A_{1}}{A_{0}} \frac{1}{\lambda}, \quad \forall \lambda \geqslant \lambda_{0} .
$$

Let

$$
\phi(\lambda)=\frac{\Phi^{\prime}(\lambda)}{\Phi(\lambda)}=\log (\Phi(\lambda))^{\prime}
$$

then we have

$$
\log \frac{\Phi\left(\lambda_{2}\right)}{\Phi\left(\lambda_{1}\right)}=\int_{\lambda_{1}}^{\lambda_{2}} \phi(t) d t
$$

From (18) it follows that

$$
\begin{aligned}
\frac{\Phi\left(\lambda_{2}\right)}{\Phi\left(\lambda_{1}\right)} & \leqslant \exp \left(\int_{\lambda_{1}}^{\lambda_{2}} \frac{3 A_{1}}{A_{0}} \frac{1}{t} d t\right) \\
& =\left(\frac{\lambda_{2}}{\lambda_{1}}\right)^{\frac{3 A_{1}}{A_{0}}}, \quad \forall \lambda_{2} \geqslant \lambda_{1} \geqslant \lambda_{0} .
\end{aligned}
$$

Now we estimate the remainder $\mathcal{R}(\tau)$ with $0<\delta<1$. Let $0<s<1$ be a number to be chosen later and write

$$
\mathcal{W}\left(\tau+\tau^{1-\delta}\right)-\mathcal{W}\left(\tau-\tau^{1-\delta}\right)=\mathcal{W}_{1}(\tau)+\mathcal{W}_{2}(\tau)
$$

where

$$
\mathcal{W}_{1}(\tau)=(2 \pi)^{-n} \int_{\substack{\tau-\tau^{1-\delta}<p+q \leqslant \tau+\tau^{1-\delta} \\ q \leqslant \tau-\tau^{1-s \delta}}} d x d \xi
$$

and

$$
\mathcal{W}_{2}(\tau)=(2 \pi)^{-n} \int_{\substack{c-\tau^{1-\delta}<+q \leqslant \tau+\tau^{1-\delta} \\ q>\tau-\tau^{1-s \delta}}} d x d \xi
$$


Let us estimate $\mathcal{W}_{1}$. On the domain of integration of $\mathcal{W}_{1}$ we have

$$
\tau+\tau^{1-\delta}-q \geqslant p>\tau-\tau^{1-\delta}-q \geqslant \tau^{1-s \delta}-\tau^{1-\delta} .
$$

Because

$$
\tau^{1-s \delta}-\tau^{1-\delta}=\tau^{1-s \delta}\left(1-\tau^{(s-1) \delta}\right) \rightarrow+\infty, \quad \text { as } \tau \rightarrow+\infty,
$$

there exists $\tau_{1}>0$ such that

$$
\tau^{1-s \delta}-\tau^{1-\delta} \geqslant \lambda_{0}, \quad \forall \tau \geqslant \tau_{1} .
$$

Then from (19) and Corollary 1] we have

$$
\begin{aligned}
(2 \pi)^{n} \mathcal{W}_{1}(\tau) & =\int_{q \leqslant \tau-\tau^{1-s \delta}}\left(\int_{\tau-\tau^{1-\delta}-q<p \leqslant \tau+\tau^{1-\delta}-q} d \xi\right) d x \\
& =\int_{q \leqslant \tau-\tau^{1-s \delta}}\left\{\Phi\left(\tau+\tau^{1-\delta}-q(x)\right)-\Phi\left(\tau-\tau^{1-\delta}-q(x)\right)\right\} d x \\
& \leqslant \int_{q \leqslant \tau-\tau^{1-s \delta}} \Phi(\tau-q)\left\{\left(1+\frac{\tau^{1-\delta}}{\tau-q}\right)^{A}-\left(1-\frac{\tau^{1-\delta}}{\tau-q}\right)^{A}\right\} d x
\end{aligned}
$$

for all $\tau \geqslant \tau_{1}$. This in turn implies that there exists $\tau_{2} \geqslant \tau_{1}$ such that

$$
\begin{aligned}
\mathcal{W}_{1}(\tau) & \prec \int_{q \leqslant \tau-\tau^{1-s \delta}} \Phi(\tau-q) \frac{\tau^{1-\delta}}{\tau-q} d x \\
& \leqslant \tau^{(s-1) \delta} \int_{q \leqslant \tau-\tau^{1-s \delta}} \Phi(\tau-q) d x \\
& =\tau^{(s-1) \delta} \mathcal{W}(\tau), \quad \forall \tau \geqslant \tau_{2} .
\end{aligned}
$$

Now we estimate $\mathcal{W}_{2}$. Because $p$ is non-negative as $|\xi| \rightarrow+\infty$, there exists $\tau_{3} \geqslant 1$ such that, on the domain of integration, we have

$$
\begin{aligned}
& p \leqslant \tau+\tau^{1-\delta}-q<\tau^{1-\delta}+\tau^{1-s \delta}=\tau^{1-s \delta}\left(\tau^{(s-1) \delta}+1\right) \leqslant 2 \tau^{1-s \delta}, \\
& q \leqslant \tau+\tau^{1-\delta}-p \leqslant 2 \tau,
\end{aligned}
$$

for $\tau \geqslant \tau_{3}$.

It follows that

$$
\begin{aligned}
\mathcal{W}_{2}(\tau) & \leqslant(2 \pi)^{-n} \int_{p(\xi) \leqslant 2 \tau^{1-s \delta}} d \xi \int_{q(x) \leqslant 2 \tau} d x \\
& =(2 \pi)^{-n} \Phi\left(2 \tau^{1-s \delta}\right) \mathcal{V}(2 \tau),
\end{aligned}
$$

for all $\tau \geqslant \tau_{3}$. Now, from (11) we have

$$
\mathcal{V}(2 \tau) \prec \mathcal{V}\left(\frac{\tau}{2}\right), \quad \forall \tau \geqslant \tau_{0} .
$$


On the other side, from (17), we have that

$$
\begin{aligned}
\Phi\left(2 \tau^{1-s \delta}\right) & =A_{0}\left(2 \tau^{1-s \delta}\right)^{a}\left(\log \left(2 \tau^{1-s \delta}\right)\right)^{b}(1+o(1)) \\
& =2^{a}(1-s \delta)^{b} A_{0} \tau^{(1-s \delta) a}(\log \tau)^{b}(1+o(1)) \\
& =2^{2 a}(1-s \delta)^{b} A_{0} \tau^{-s \delta a}\left(\frac{\tau}{2}\right)^{a}\left(\log \left(\frac{\tau}{2}\right)\right)^{b}(1+o(1)) \\
& =2^{2 a}(1-s \delta)^{b} \tau^{-s \delta a} \Phi\left(\frac{\tau}{2}\right)(1+o(1)), \quad \text { for } \tau \rightarrow+\infty .
\end{aligned}
$$

But these estimates imply that there exists $\tau_{4} \geqslant \tau_{0}$ such that

$$
\begin{aligned}
\mathcal{W}_{2}(\tau) & \prec \tau^{-s \delta a} \Phi\left(\frac{\tau}{2}\right) \mathcal{V}\left(\frac{\tau}{2}\right) \\
& \leqslant \tau^{-s \delta a} \int_{p \leqslant \tau / 2,} d x d \xi \\
& \leqslant \tau^{-s \delta a} \mathcal{W}(\tau), \quad \forall \tau \geqslant \tau_{4} .
\end{aligned}
$$

Eventually, from (21) and (22), we have that it suffices to choose $s$ such that

$$
(s-1) \delta=-s \delta a,
$$

that is,

$$
s=\frac{1}{1+a},
$$

in order to obtain

$$
\mathcal{W}\left(\tau+\tau^{1-\delta}\right)-\mathcal{W}\left(\tau-\tau^{1-\delta}\right)=O\left(\tau^{-\epsilon}\right) \mathcal{W}(\tau), \quad \text { as } \tau \rightarrow+\infty,
$$

with

$$
\epsilon=\frac{\delta a}{1+a}
$$

\section{An example}

We end our paper by giving an example of potential verifying our hypotheses. First we prove the following

Proposition 1. Let $q: \mathbb{R}^{n} \rightarrow \mathbb{R}$ be a smooth function such that there exist $\mu \in \mathbb{R}_{+}^{n}$, $C_{\mu}>1$ and $R_{0} \geqslant 0$ such that

$$
q(\mu \cdot x) \geqslant C_{\mu} q(x), \quad \forall|x| \geqslant R_{0},
$$

where

$$
\mu \cdot x=\sum_{i=1}^{n} \mu_{i} x_{i} .
$$

Let $N$ be the smallest integer such that

$$
C_{\mu}^{N} \geqslant 2
$$

Then $\mathcal{V}$ satisfies condition (11) with

$$
\tau_{0}=\frac{C_{\mu}^{N}}{2} \max _{|x| \leqslant R_{0}} q(x) .
$$


Proof. Letting $x=\mu \cdot y$ and using (23) yields

$$
\begin{aligned}
\int_{q(x) \leqslant 2 \tau} d x & =\mu_{1} \cdots \mu_{n} \int_{q(\mu \cdot y) \leqslant 2 \tau} d y \\
& \leqslant \mu_{1} \cdots \mu_{n}\left\{\int_{|y|<R_{0}} d y+\int_{\substack{q(\mu \cdot y) \leqslant 2 \tau \\
|y| \geqslant R_{0}}} d y\right\} \\
& \leqslant \mu_{1} \cdots \mu_{n}\left\{\int_{|y|<R_{0}} d y+\int_{\substack{C_{\mu} q(y) \leqslant 2 \tau \\
|y|>R_{0}}} d y\right\} \\
& \leqslant \mu_{1} \cdots \mu_{n} \int_{q(y) \leqslant 2 C_{\mu}^{-1} \tau} d y,
\end{aligned}
$$

for $\tau \geqslant \tau_{0}$. By iterating, we obtain

$$
\mathcal{V}(2 \tau)=\int_{q(x) \leqslant 2 \tau} d x \leqslant\left(\mu_{1} \cdots \mu_{n}\right)^{N} \int_{q(x) \leqslant 2 C_{\mu}^{-N} \tau} d x \leqslant\left(\mu_{1} \cdots \mu_{n}\right)^{N} \mathcal{V}(\tau),
$$

for all $\tau \geqslant \tau_{0}$.

Now we can construct a class of operators of Schrödinger type, with potential verifying condition (11).

Proposition 2. Let us consider

- a real polynomial $p(\xi)$ vanishing at the origin and hypoelliptic, i.e. such that

$$
\lim _{|\xi| \rightarrow+\infty} p(\xi)=+\infty
$$

and

$$
\nabla p(\xi) \prec p(\xi)^{1-\rho}, \quad \text { for } p(\xi) \geqslant 1,
$$

with $0<\rho \leqslant 1$,

- a positive smooth function $q(x)$ such that

- $p(\xi)+q(x)>0, \forall(x, \xi) \in \mathbb{R}^{n} \times \mathbb{R}^{n}$,

- there exists $0 \leqslant \nu<\rho$ such that for all $\alpha \in \mathbb{N}^{n}$ we have $D^{\alpha} q(x) \prec$ $q(x)^{1+\nu|\alpha|}, \forall x \in \mathbb{R}^{n}$,

- it satisfies the hypothesis of Proposition 1 .

Then $h^{w}=p^{w}+q$ satisfies the hypotheses of Theorem 3 .

Proof. We give a brief sketch of the proof. By Proposition 11 condition (11) is satisfied.

Then it suffices to verify the hypotheses of Theorem 1 Consider the metric

$$
g_{x, \xi}(y, \eta)=h(x, \xi)^{2 \nu}|y|^{2}+h(x, \xi)^{-2 \rho}|\eta|^{2} .
$$

Then one verifies that $g$ and $h$ are locally $\phi$ temperate; for example see [3]. Moreover one can show that $h \rightarrow+\infty$, as $|x|+|\xi| \rightarrow+\infty, h \in S(h, g)$ and (3) is verified. We omit the details. 
For example we can take (see [1], section 1.1)

$$
\begin{aligned}
& p(\xi)=\left(\xi_{1}^{2 k}-\xi_{2}^{2 k-1}\right)^{2}+\xi_{1}^{2 k} \xi_{2}^{2 k-2}, \\
& q(x)=\exp \left(x_{1}^{2 m_{1}}+x_{2}^{2 m_{2}}\right),
\end{aligned}
$$

where $k$ is an integer greater than 1 and $m_{1}$ and $m_{2}$ are positive integers.

\section{REFERENCES}

[1] Boggiatto, P., Buzano, E. and Rodino, L., Global hypoellipticity and spectral theory, Mathematical Research, vol. 92, Akademie Verlag, Berlin, 1996. MR 97m:35040

[2] Buzano, E., Some remarks on the Weyl asymptotics by the approximate spectral projection method, Boll. U.M.I. 3-B (2000), no. 8, 775-792 MR 2001k:35232

[3] Buzano, E. and Ziggioto, A., Weyl formula for multi-quasi-elliptic operators of Schrödinger type, Ann. Mat. Pura Appl. 180 (2001), 223-243. MR 2002f:35179

[4] Buzano, E. and Nicola, F., Hypoelliptic symbols and complex powers of pseudodifferential operators in the Weyl-Hörmander classes, in preparation.

[5] Dencker, N., The Weyl calculus with locally temperate metrics and weights, Arkiv för Mat. 24 (1986), 59-79. MR 87m:47111

[6] Hörmander, L., The Weyl calculus of pseudo-differential operators, Comm. Pure Appl. Math. 32 (1979), 359-443. MR 80j:47060

[7] $\_$On the asymptotic distribution of the eigenvalues of pseudodifferential operators in $\mathbb{R}^{n}$, Arkiv för Mat. 17 (1979), 297-313. MR 82i:35140

[8] — The analysis of linear partial differential operators II, Grundleheren der mathematischen Wissenschaften, vol. 257, Springer-Verlag, Berlin, 1983. MR 85g:35002b

[9] Nilsson, N., Monodromy and asymptotic properties of certain multiple integrals, Arkiv för Mat. 18 (1980), 181-198. MR 83i:32020

[10] Shubin, M. A., Pseudodifferential operators and spectral theory, Springer-Verlag, Berlin, 1987. MR 88c: 47105

[11] Taylor M. E., Pseudodifferential Operators, Princeton Mathematical Series, vol. 34, Princeton University Press, Princeton, New Jersey, 1981. MR 82i:35172

Dipartimento di Matematica, Università di Torino, Via Carlo Alberto 10, 10123 TORINO, ITALIA

E-mail address: buzano@dm.unito.it

Dipartimento di Matematica, Università di Torino, Via Carlo Alberto 10, 10123 TORINO, ITALIA

E-mail address: ziggioto@dm.unito.it 